\title{
Bioactivity of Citrus essential oils (CEOs) against microorganisms associated with spoilage of some fruits
}

\author{
Oluwatayo Benjamin Ajayi-Moses ${ }^{1}$, Clement Olusola Ogidi ${ }^{1,2^{*}}$ (D) and Bamidele Juliet Akinyele ${ }^{1}$
}

\begin{abstract}
Background: This study evaluated the antimicrobial potentials of Citrus essential oils (CEOs) against spoilage microorganisms isolated from selected fruits. The fruits were randomly purchased from different markets in Akure, Nigeria.

Methods: The microorganisms were isolated and identified using molecular tools. In vitro antimicrobial efficacies of CEOs and their synergistic potentials were tested against spoilage microorganisms using agar well diffusion. The bioactive compounds in CEOs were identified using gas chromatography-mass spectrometry (GC-MS).

Results: The highest bacterial count $\left(5.84 \times 10^{5} \mathrm{cfu} / \mathrm{g}\right)$ was recorded in tomatoes, while African star apple had the highest fungal count of $3.04 \times 10^{5} \mathrm{sfu} / \mathrm{g}$. Microorganisms isolated from fruits were Bacillus spp., Micrococcus /uteus, Serratia marcescens, Aspergillus spp., Mucor piriformis, Fusarium oxysporum, Penicillium spp., Rhizopus spp., Alternaria alternata and others. Phytochemicals in the CEOs were anthraquinones, cardiac glycosides, tannins, alkaloids, terpenoids, saponins, steroids, flavonoids and phenol. The diameter zones of inhibition displayed by CEOs against tested microorganisms at $100 \mathrm{mg} / \mathrm{ml}$ ranged from $3.3 \mathrm{~mm}$ to $26.8 \mathrm{~mm}$ with B. muralis being the most susceptible bacteria. The minimum inhibitory concentration (MIC) against all the tested isolates ranged from 12.5 to $100 \mathrm{mg} / \mathrm{ml}$, while the minimum bactericidal and fungicidal concentrations ranged from 25 to $\geq 100 \mathrm{mg} / \mathrm{ml}$. The synergism between lime and lemon at ratio 1:1 had better antimicrobial activity than each essential oil when used singly. GC-MS revealed the presence of limonene, beta-pinene, alpha-phellandrene, terpinen-4-ol, alpha-terpineol and geraniol in EOs of lime and lemon.
\end{abstract}

Conclusion: The inhibitory potential of CEOs could be attributed to their bioactive compounds, which can be exploited and used as preservatives by food industries.

Keywords: Rotten fruit, Post-harvest, Fruit peels, Limonene and preservation

\section{Background}

The relevance of fruits to human diet cannot be overemphasized because of their nutritional values and various benefits [1]. Fruits are source of minerals, vitamins and other essential growth factors that are needed for body metabolism. The nutritional contents of fruits vary, thereby making some of them to be highly demanded than others [2]. However, annual reports

\footnotetext{
*Correspondence: clementogidi@yahoo.com

${ }^{2}$ Biotechnology Unit, Department of Biological Sciences, Kings University, P.M.B. 555, Odeomu, Nigeria

Full list of author information is available at the end of the article
}

have shown that 30 to $50 \%$ of fruits produced do not get to the final consumers due to spoilage, especially during the post-harvest stage [3, 4]. Substantial losses incurred during this stage are slight loss in organoleptic quality to total spoilage [5]. This affects the shelf life as well as the market value of the fruits. The losses occurred as a result of spoilage associated with microorganisms, especially fungi known to produce mycotoxins such as aflatoxin, patulin and ochratoxin, which are secondary metabolites that are harmful to humans and animals [6].

The means to control post-harvest spoilage of fruits and fruit products is mainly by using preservative agents. 
Despite the benefits of chemical preservatives, their persistent use has raised some public health concerns as regard their safety and possible detrimental effect on nutritional composition and organoleptic parameters of foods [7]. More so, the use of antibiotics by food industries as preservative agents has contributed largely to the global challenge of antibiotic resistance [8]. Therefore, food industries are currently in pursuit of adopting natural bioactive in plants as alternative preservatives to prolong shelf life of food products and to ensure the safety of consumers. The use of plant extracts as better alternative to chemical antimicrobials in the management of post-harvest spoilage of fruit is being researched on, thereby reducing incidence of multiple drug resistance and safeguarding food safety [9]. The peel of Citrus fruits such as orange, lemon, grapefruit, pomelo and lime is rich sources of essential oils. These Citrus essential oils (CEOs) contain various phytochemicals that have been reported to be effective against pathogenic microorganisms [10]. This study is therefore designed to evaluate the inhibitory activities of EOs from lemon (Citrus limon), grapefruit (C. paradisi) and lime (C. aurantifolia) against selected microorganisms associated with the spoilage of various fruits.

\section{Materials and methods Collection of fruit samples}

Lime, lemon and grape fruits used as sources of essential oils were obtained from a farm at Ogbese, Ondo State. A total of 270 fruits, 30 each of pawpaw, apple, pineapple, watermelon, banana, orange, cucumber, tomato and African star apple were obtained from different locations (markets/depots) in Akure. All the samples collected were separately placed in a sterile polythene bags and labelled appropriately, before transported to Microbiology laboratory, The Federal University of Technology, Akure (FUTA), for the microbial analysis. The authentication of each fruit was carried out at the Department of Crop, Soil and Pest Management of the University.

\section{Isolation of microorganisms}

Fruits were surface sterilized with $70 \% \mathrm{v} / \mathrm{v}$ alcohol, $1 \% \mathrm{v} / \mathrm{v}$ sodium hypochlorite to eliminate the normal microflora of the fruits and then rinsed three times in sterile distilled water. Segments of tissues from the margins of the rotten areas were aseptically cut and transferred into sterilized peptone water $(20 \mathrm{ml})$. The specimen was shaken vigorously and then allowed to stand for $30 \mathrm{~min}$. From this, tenfold serial dilutions were done, and an aliquot of $0.1 \mathrm{ml}$ of $10^{5}$ was then aseptically transferred into Petri dish and molten nutrient agar was introduced. The plate was gently swirled and allowed to solidify at room temperature, after which the plates were incubated at $37^{\circ} \mathrm{C}$ for $24 \mathrm{~h}$. For the isolation of associated fungi, $0.1 \mathrm{ml}$ aliquot from $10^{4}$ was aseptically dispensed into Petri dishes, and thereafter, sterilized molten potato dextrose agar (PDA) was transferred into the Petri dish and incubated for 2-3 days at $25{ }^{\circ} \mathrm{C}$. Discrete colonies were observed, counted, recorded as colony forming unit per gram $(\mathrm{cfu} / \mathrm{g})$ for bacteria and spore forming unit per gram (sfu/g) for fungi.

\section{Identification of isolated microorganisms}

Colonies with unique morphological appearance were selected from the plates. A single pure colony was obtained by subculture [11]. Isolates were tentatively grouped according to their morphological, cultural and staining characteristics. Biochemical tests carried out include: catalase test, production of hydrogen sulphide $\left(\mathrm{H}_{2} \mathrm{~S}\right)$, indole, urease, methyl red, oxidase, coagulase, motility, methyl red, Voges-proskauer, starch hydrolysis and sugars fermentation. The biochemical results were compared with Bergey's Manual of Systematic Bacteriology [12]. The isolated fungi were identified using cultural and morphological characteristics [13, 14], and microscopic observations were interpreted according to Barnett et al. [15]. Standard antibiotic susceptibility profiles were used to select bacteria and yeast for molecular studies and used as indicator microorganisms.

\section{Molecular identification of bacterial and yeast isolates}

Single colony of bacteria and yeasts was grown in Luria Bertain medium for $24 \mathrm{~h}$. Extraction of DNA was done according to the method described by Zolan and Pukkila [16]. $16 \mathrm{~S}$ universal primers were used to amplify $16 \mathrm{~S}$ rRNA gene of bacteria, while ITS primer was used for yeast gene amplification. The PCR mix comprised of $1 \mu \mathrm{l}$ of $10 \times$ buffer, $0.4 \mu \mathrm{l}$ of $50 \mathrm{mM} \mathrm{MgCl} 2,0.5 \mu \mathrm{l}$ of $2.5 \mathrm{mM}$ dNTPs, $0.5 \mu \mathrm{l}$ of $5 \mathrm{mM}$ forward and reverse primers, $0.05 \mu \mathrm{l}$ of 5 units/ $\mu \mathrm{l}$ Taq with $2 \mu \mathrm{l}$ of template DNA and $5.05 \mu \mathrm{l}$ of distilled water to make-up $10 \mu \mathrm{l}$ reaction mix. The PCR profile used was initially denatured at $94{ }^{\circ} \mathrm{C}$ for $3 \mathrm{~min}$, followed by 30 cycles of $94{ }^{\circ} \mathrm{C}$ for $60 \mathrm{~s}, 56{ }^{\circ} \mathrm{C}$ for $60 \mathrm{~s}, 72{ }^{\circ} \mathrm{C}$ for $120 \mathrm{~s}$ and the final extension temperature of $72{ }^{\circ} \mathrm{C}$ for $5 \mathrm{~min}$ and the $10{ }^{\circ} \mathrm{C}$ hold forever. A $5 \mu \mathrm{l}$ of PCR products then visualized by electrophoresis in $1.5 \%$ agarose (Bioline) in $1 \times$ TAE buffer for $25 \mathrm{~min}$ at $100 \mathrm{~V}$. The amplicons were purified before the sequencing using $2 \mathrm{M}$ sodium acetate wash techniques. Sequencing of PCR products was performed. The full-length gene sequences were compiled and compared with those from the National Centre of Biotechnology Information (NCBI) using the Basic Local Alignment Search Tool (BLAST) program. 


\section{Extraction of Citrus essential oils}

Essential oils were extracted from the Citrus rind by the process of steam distillation for $5 \mathrm{~h}$ using a Clevenger apparatus [17]. The volume of CEOs was determined from a calibrated trap. The EOs in the distillate were dried over anhydrous $\mathrm{Na}_{2} \mathrm{SO}_{4}$ and kept in the freezer. The EOs was extracted from collected material by hydrodistillation for $5 \mathrm{~h}$ using Clevenger-type apparatus [18]. A clear, light yellow coloured, oily layer was obtained on the top of the aqueous distillate, which was separated from the latter and dried with anhydrous sodium sulphate. The extracted EOs were kept in air-tight sealed glass vials covered with aluminium foil at $4{ }^{\circ} \mathrm{C}$ until further analysis.

\section{Qualitative phytochemical analysis of Citrus essential oils} Qualitative and quantitative phytochemicals in CEOs were determined using the standard methods reported by Trease and Evans [19], Harborne and Baxter [20]. The bioactive compounds in the most effective CEOs were determined by gas chromatography-mass spectrometry (GC-MS). Briefly, GC-MS (QP2010 plus Shimadzu, Japan) was equipped with a split injector, an ion-trap mass spectrometer detector and a fused-silica capillary column having a thickness of $1.00 \mu \mathrm{m}$, dimensions of $0.32 \mathrm{~mm} \times 30 \mathrm{~m}$ and temperature limits of $60{ }^{\circ} \mathrm{C}$ to $325{ }^{\circ} \mathrm{C}$. The column temperature was programmed between 60 and $240{ }^{\circ} \mathrm{C}$ at a rate of $0.5 \mathrm{~m} / \mathrm{s}$ with pressure of $100.2 \mathrm{Kpa}$. The temperature of the injector and detector was at $250{ }^{\circ} \mathrm{C}$ and $200{ }^{\circ} \mathrm{C}$, respectively. Helium gas was used as a carrier gas at flow rate of $0.46 \mathrm{~m} / \mathrm{s}$. The MS analysis was done based on comparative retention times, mass and peaks of chemical compounds using the computer-aided matching of unknown mass spectra of compounds with the known compounds stored in the software database library from the National Institute of Standards (NIS), Washington, USA, having more than 62,000 patterns as the reference database. This library enables the facilitation of comparison of generated spectra with the standards using probability-based matching algorithms (PBMA). The GC-MS had been pre-fitted with a set of automated internal validity programmes for the analysis, including the adjustment of retention time function, scan measurement, quick and accurate compound identification from chromatogram with search based on mass spectra similarity and other quality. The name, molecular weight and the structure of the components of the tested materials were ascertained with database library from the NIS, Washington, USA.

\section{In vitro antimicrobial activity of CEOs}

The antimicrobial efficiency of the CEOs against the spoilage microorganisms isolated from various fruits was carried out using agar well diffusion [21]. Briefly, suspension of test microorganisms was adjusted with the aid of spectrophotometer (UNICO 1100 RS) to 0.5 McFarland standard according to the method of Clinical and Laboratory Standards Institute CLSI [22]. Sterile cotton swabs were dipped in the microbial suspension and evenly spread over the entire surface of the agar plate to obtain uniform inoculums. Well in plate was made using a sterilized cork borer. Prior to this assay, the extract was sterilized by passing through a Millipore membrane filter $(0.22 \mu \mathrm{m})$. The sterility of extract was further confirmed after Millipore filtration, by introducing $0.1 \mathrm{ml}$ of supposed sterile extract into sterilized nutrient agar and potato dextrose agar. The plates were incubated at $37^{\circ} \mathrm{C}$ for bacteria and $25^{\circ} \mathrm{C}$ for fungi. Concentration $(100 \mathrm{mg} /$ $\mathrm{ml}$ ) of CEOs was obtained by reconstituting with DMSO (2\%). Each CEO (50 $\mu \mathrm{l})$ was dispensed in respective well using micropipette. Chloramphenicol and nystatin were used as positive controls against bacteria and fungi, respectively, while DMSO (2\%) was used as the negative control. All the plates were labelled appropriately and incubated at $37^{\circ} \mathrm{C}$ for $24 \mathrm{~h}$ for bacteria and at $25^{\circ} \mathrm{C}$ for $48 \mathrm{~h}$ for fungi. Each test was carried out in replicates. The diameter of inhibited zones around wells was measured in millimetre $(\mathrm{mm})$. The synergistic effect of lime, lemon and grapefruit essential oils (1:1 v:v and 1:1:1 v:v:v) against isolated microorganisms was also assessed using the method described above.

Determination of minimum inhibitory concentration (MIC), minimum bactericidal concentration (MBC) and minimum fungicidal concentration (MFC)

The MIC, MBC and MFC of CEOs against the microbial isolates were determined using the guidelines of CLSI [22] The CEOs were reconstituted using 2\% v/v DMSO. The varying concentrations (100, 75 50, 25, 12.5, $6.25 \mathrm{mg} /$ $\mathrm{ml}$ ) of the extracts were prepared and incorporated into a set of sterile tubes. Each tube was then inoculated with $0.1 \mathrm{ml}$ of standardized test organism and incubated for $24 \mathrm{~h}$ and $48 \mathrm{~h}$ for bacteria and fungi, respectively. From each tube, $0.5 \mathrm{ml}$ was plated on nutrient agar and potato dextrose agar using pour plate technique to enumerate the viable count after incubation. The MICs were recorded as the lowest concentration of CEOs preventing growth of macroscopically visible colonies on plates at the time when there was visible growth on oil-free plates. To determine the MBC and MFC, further incubation was done for 24 and $48 \mathrm{~h}$ for bacteria and fungi, respectively, and the concentration at which no visible growth was seen after the further incubation was noted as the MBC and MFC. 


\section{Results}

\section{Total microbial load of selected fruits}

Table 1 shows the total microbial count from each fruit collected from various depots. The highest bacterial count $\left(5.84 \times 10^{5} \mathrm{cfu} / \mathrm{g}\right)$ was recorded in tomatoes collected from King's market, while apple fruits had no bacterial growth. African star apple from Owena market had the highest fungal count of $3.04 \times 10^{5} \mathrm{sfu} / \mathrm{g}$, while pawpaw obtained from FUTA Southgate market axis had the least fungal count of $1.10 \times 10^{4} \mathrm{sfu} / \mathrm{g}$. The total fungal count for the apparently healthy fruits ranged between 0.20 and $1.87 \times 10^{2} \mathrm{sfu} / \mathrm{g}$, but there was no bacterial growth on all apparently healthy fruits.

\section{Types and distribution of microorganisms isolated from spoilt fruits}

Bacteria, yeast and mould were isolated from the spoilt pawpaw, apple, pineapple, watermelon, orange, cucumber, tomato, banana and African star apple (Table 2ac). The bacteria isolated were Lactobacillus plantarum, Bacillus cereus, Bacillus subtilis, Micrococcus luteus, Streptococcus uberis, Proteus vulgaris and Serratia marcescens, while fungi isolates include Aspergillus niger, A. flavus, A. fumigatus, A. parasiticus, Mucor piriformis, $M$. racemosis, Fusarium oxysporum, $F$. avenaceum, F. solani, Penicillium digitatum, P. expansum, Rhizopus stolonifer, R. oryzae, Alternaria alternata, Saccharomyces cerevisiae, Candida tropicalis, $C$. krusei, Kluyveromyces marxianus, Torulopsis fragaria, Pichia kluyveri, P. fermentans, P. anomala, Geotrichum candidum, Zygosaccharomyces rouxii and Z. bailii. Lactobacillus plantarum was the most predominant bacterium with value of $23 \%$, while Aspergillus niger was a fungus with the highest manifestation in all the fruits with frequency of $24.4 \%$. Other species of Aspergillus had a combined frequency of $21.7 \%$. Fusarium species had combined occurrence of $29.1 \%$ and were present from all fruits except apple, while Erwinia cacticida and Alternaria alternata were the least encountered bacterium and fungus with values of 2.6 and $1.9 \%$, respectively. Molecular identification of the bacterial and fungal isolates is shown in Table 3. The sequence obtained was analysed with Basic Local Alignment Search Tool (BLAST) in National Centre for Biotechnology Information (NCBI) database.

\section{Phytochemicals and bioactive compound in CEOs}

The quantity of phytochemicals in CEOs is shown in Fig. 1. Phenol, flavonoids, alkaloids, steroids and

Table 1 Microbial count from fruits collected from different locations

\begin{tabular}{|c|c|c|c|c|c|c|}
\hline Fruits & FSG & OF & OJM & owM & CFS & $\mathrm{AHF}$ \\
\hline \multicolumn{7}{|c|}{ Bacteria count $\times 10^{5} \mathrm{cfu} / \mathrm{g}$} \\
\hline Pawpaw & $1.73 \pm 0.05^{b}$ & $0.54 \pm 0.07^{a b}$ & $2.35 \pm 0.09^{b}$ & $3.26 \pm 0.07^{b}$ & $2.47 \pm 0.27^{b}$ & $0.00 \pm 0.00^{a}$ \\
\hline Apple & $0.00 \pm 0.00^{\mathrm{a}}$ & $0.00 \pm 0.00^{\mathrm{a}}$ & $0.00 \pm 0.00^{a}$ & $0.00 \pm 0.00^{\mathrm{a}}$ & $0.00 \pm 0.00^{\mathrm{a}}$ & $0.00 \pm 0.00^{\mathrm{a}}$ \\
\hline Pineapple & $2.86 \pm 0.09^{d}$ & $0.81 \pm 0.21^{b c}$ & $2.58 \pm 0.35^{b}$ & $3.08 \pm 0.25^{b}$ & $3.55 \pm 0.13^{c}$ & $0.00 \pm 0.00^{\mathrm{a}}$ \\
\hline Watermelon & $3.83 \pm 0.11^{e}$ & $1.16 \pm 0.03^{\mathrm{cd}}$ & $4.30 \pm 0.28^{c}$ & $4.32 \pm 0.60^{c}$ & $5.26 \pm 0.15^{e}$ & $0.00 \pm 0.00^{\mathrm{a}}$ \\
\hline Tomato & $5.37 \pm 0.15^{\mathrm{g}}$ & $1.70 \pm 0.23^{d}$ & $5.84 \pm 0.43^{d}$ & $5.00 \pm 0.30^{c d}$ & NA & $0.00 \pm 0.00^{\mathrm{a}}$ \\
\hline Cucumber & $4.70 \pm 0.13^{f}$ & $1.04 \pm 0.21^{b c}$ & $4.79 \pm 0.26^{c}$ & $5.48 \pm 0.49^{d}$ & $4.32 \pm 0.23^{d}$ & $0.00 \pm 0.00^{\mathrm{a}}$ \\
\hline Orange & $2.22 \pm 0.05^{c}$ & $0.47 \pm 0.20^{a b}$ & $2.23 \pm 0.07^{b}$ & $2.78 \pm 0.38^{b}$ & $2.11 \pm 0.12^{b}$ & $0.00 \pm 0.00^{\mathrm{a}}$ \\
\hline Banana & $2.22 \pm 0.14^{c}$ & $1.18 \pm 0.30^{\mathrm{cd}}$ & $2.88 \pm 0.48^{b}$ & $3.14 \pm 0.24^{b}$ & $2.35 \pm 0.19 b$ & $0.00 \pm 0.00^{a}$ \\
\hline African star apple & $5.54 \pm 0.16^{g}$ & $1.34 \pm 0.17^{\mathrm{cd}}$ & $4.90 \pm 0.42^{c}$ & $4.38 \pm 0.03^{c}$ & NA & $0.00 \pm 0.00^{\mathrm{a}}$ \\
\hline \multicolumn{7}{|c|}{ Fungal count $\times 10^{4} \mathrm{sfu} / \mathrm{g}^{\#}$} \\
\hline Pawpaw & $1.10 \pm 0.06^{\mathrm{a}}$ & $2.73 \pm 0.17^{a b}$ & $4.60 \pm 0.38^{b}$ & $4.07 \pm 0.37^{\mathrm{a}}$ & $2.20 \pm 0.31^{a b}$ & $0.47 \pm 0.03^{b}$ \\
\hline Apple & $5.03 \pm 1.08^{b}$ & $5.40 \pm 0.35^{c}$ & $2.93 \pm 0.18^{a}$ & $2.03 \pm 0.20^{\mathrm{a}}$ & $2.93 \pm 0.24^{b}$ & $0.23 \pm 0.03^{\mathrm{a}}$ \\
\hline Pineapple & $1.97 \pm 0.27^{\mathrm{a}}$ & $5.53 \pm 0.34^{c}$ & $3.43 \pm 0.26^{a b}$ & $9.53 \pm 0.41^{b}$ & $7.13 \pm 0.41^{d}$ & $0.60 \pm 0.06^{b c}$ \\
\hline Watermelon & $3.07 \pm 0.77^{a b}$ & $2.87 \pm 0.33^{\mathrm{ab}}$ & $3.03 \pm 0.23^{\mathrm{a}}$ & $4.23 \pm 0.15^{\mathrm{a}}$ & $5.13 \pm 0.18^{c}$ & $0.90 \pm 0.06^{d}$ \\
\hline Tomato & $3.27 \pm 0.43^{\mathrm{ab}}$ & $10.03 \pm 0.49^{d}$ & $8.63 \pm 0.35^{c}$ & $3.57 \pm 0.66^{\mathrm{a}}$ & $2.57 \pm 0.43^{a b}$ & $1.23 \pm 0.03^{\mathrm{e}}$ \\
\hline Cucumber & $13.27 \pm 1.62^{c}$ & $13.27 \pm 0.41^{\mathrm{e}}$ & $17.13 \pm 0.67^{d}$ & $9.03 \pm 0.44^{b}$ & $10.87 \pm 0.64^{e}$ & $0.63 \pm 0.03^{c}$ \\
\hline Orange & $1.57 \pm 0.03^{\mathrm{a}}$ & $1.37 \pm 0.08^{\mathrm{a}}$ & $2.27 \pm 0.13^{\mathrm{a}}$ & $1.87 \pm 0.09^{a}$ & $3.00 \pm 0.15^{b}$ & $0.27 \pm 0.03^{a}$ \\
\hline Banana & $2.40 \pm 0.87^{a}$ & $3.97 \pm 0.10^{b c}$ & $3.47 \pm 0.24^{\mathrm{ab}}$ & $3.27 \pm 0.75^{a}$ & $1.10 \pm 0.21^{\mathrm{a}}$ & $0.20 \pm 0.06^{a}$ \\
\hline African star apple & $29.03 \pm 0.75^{d}$ & $20.17 \pm 0.81^{f}$ & $22.07 \pm 1.07^{\mathrm{e}}$ & $30.40 \pm 2.94^{c}$ & $12.27 \pm 1.22^{\mathrm{e}}$ & $1.87 \pm 0.07^{f}$ \\
\hline
\end{tabular}

Data are represented as mean \pm standard error $(n=3)$ with the same superscript down the column are not significantly different $(p<0.05)$

FSG fruit from FUTA south gate, OF fruit from farm, OJM fruit from Ojaoba Akure, OWM fruit from Owena market, $C F S$ cathedral fruit shop, AHF apparently healthy fruit, $N A$ not available

\# Fungal count for AHF $\times 10^{2} \mathrm{sfu} / \mathrm{g}$ 
Table 2 Occurrence of mould, yeast, bacterial isolates and the fruits affected

\begin{tabular}{|c|c|c|c|c|c|c|c|c|c|c|c|}
\hline & A & B & $\mathrm{C}$ & D & $E$ & $F$ & G & $\mathrm{H}$ & 1 & $\mathrm{~N}$ & $\begin{array}{l}\text { Occurrence } \\
\text { (\%) }\end{array}$ \\
\hline \multicolumn{12}{|l|}{ (a) Mould isolates } \\
\hline Aspergillus niger & + & + & + & + & + & + & + & + & + & 132 & 24.4 \\
\hline Fusarium avenaceum & + & - & - & + & + & + & + & - & + & 94 & 17.4 \\
\hline Aspergillus flavus & + & + & + & + & + & - & + & - & + & 52 & 9.6 \\
\hline Fusarium solani & + & - & + & + & + & + & + & + & - & 44 & 8.2 \\
\hline Penicillium digitatum & + & + & - & + & + & + & + & - & + & 38 & 7.0 \\
\hline Aspergillus parasiticus & + & + & - & - & + & + & + & - & + & 37 & 6.9 \\
\hline Aspergillus fumigatus & + & - & + & - & + & + & + & - & - & 28 & 5.2 \\
\hline Penicillium expansum & - & + & + & - & + & + & + & - & + & 26 & 4.8 \\
\hline Rhizopus stolonifer & + & - & - & - & + & - & + & + & - & 21 & 3.9 \\
\hline Fusarium oxysporum & + & - & + & + & + & - & - & + & - & 19 & 3.5 \\
\hline Rhizopus oryzae & + & - & - & + & + & - & - & - & - & 15 & 2.8 \\
\hline Mucor racemosis & + & - & - & - & - & + & + & - & + & 13 & 2.4 \\
\hline Mucor piriformis & - & + & + & - & - & + & + & - & + & 11 & 2.0 \\
\hline Alternaria alternata & - & - & - & - & + & - & - & - & - & 10 & 1.9 \\
\hline \multicolumn{12}{|l|}{ (b) Yeast isolates } \\
\hline Saccharomyces cerevisiae & + & + & + & + & - & + & + & + & + & 88 & 29.4 \\
\hline Candida tropicalis & + & + & + & + & + & + & + & + & + & 49 & 16.3 \\
\hline Candida krusei & + & - & - & + & - & + & + & - & + & 38 & 12.7 \\
\hline Geotrichum candidum & - & - & - & - & + & - & + & + & - & 30 & 10.0 \\
\hline Pichia kluyveri & + & - & + & + & + & + & + & + & - & 28 & 9.3 \\
\hline Pichia fermentans & + & + & - & + & + & + & + & - & + & 21 & 7.0 \\
\hline Pichia anomala & + & + & - & - & + & + & + & - & + & 17 & 5.7 \\
\hline Torulopsis fragaria & + & + & + & + & + & - & + & - & + & 10 & 3.3 \\
\hline Zygosaccharomyces rouxii & + & - & + & - & - & + & + & - & - & 9 & 3.0 \\
\hline Zygosaccharomyces bailii & - & + & + & - & + & + & + & - & + & 7 & 2.3 \\
\hline Kluyveromyces marxianus & + & - & + & + & + & - & - & + & - & 3 & 1.0 \\
\hline \multicolumn{12}{|l|}{ (c) Bacterial isolates } \\
\hline Lactobacillus plantarum & + & - & + & + & + & + & + & + & + & 62 & 23.0 \\
\hline Bacillus cereus & + & - & - & - & + & + & - & - & + & 49 & 18.0 \\
\hline Micrococcus luteus & + & - & - & - & - & - & + & - & - & 38 & 14.1 \\
\hline Staphylococcus aureus & + & - & + & + & - & - & + & - & + & 32 & 11.9 \\
\hline Streptococcus pyogenes & - & - & - & - & - & + & - & + & - & 22 & 8.1 \\
\hline Bacillus subtilis & + & - & + & - & - & + & - & + & + & 21 & 7.8 \\
\hline Pseudomonas aeruginosa & + & - & - & + & - & - & + & + & - & 16 & 6.0 \\
\hline Proteus vulgaris & - & - & -- & - & + & - & - & - & - & 12 & 4.4 \\
\hline Serratia marcescens & - & - & - & - & - & + & - & - & + & 11 & 4.1 \\
\hline Erwinia cacticida & - & - & - & + & + & - & - & - & - & 7 & 2.6 \\
\hline
\end{tabular}

A-pawpaw, B-apple, C-pineapple, D-watermelon, E-tomato, $\mathrm{F}$-cucumber, $\mathrm{G}$-orange, $\mathrm{H}$-banana, I-African star apple, $\mathrm{N}$-number of isolates

cardiac glycosides were present in all the samples, while anthraquinones, tannin and terpenoids were absent in lemon and grapefruit EOs. Phenol had the highest values in all the three samples with $26.97,14.05$ and $10.95 \mathrm{mg} / 100 \mathrm{~g}$ in lime, lemon and grapefruit, respectively, followed by flavonoids with values ranging between 5.30 and $18.30 \mathrm{mg} / 100 \mathrm{~g}$. Figures 2 and 3 shows chromatogram peaks for each bioactive compounds in CEOs, while Table 4 shows the peak area, molecular weight of bioactive compounds identified by GC-MS. Limonene, alpha-terpineol, beta-pinene, terpinen-4-ol, alpha-phellandrene and others were present in CEOs. 
Table 3 Molecular identities of bacteria and yeast isolated from fruits

\begin{tabular}{|c|c|c|c|}
\hline Microorganisms $^{\mathrm{a}}$ & BLAST identity & GenBank accession no & Closeness \% \\
\hline \multicolumn{4}{|l|}{ Bacteria } \\
\hline Leuconostoc lactis & Lactobacillus plantarum strain KLDS1 & EU626013 & 98 \\
\hline Bacillus cereus & Bacillus muralis strain KmS1200907 & MG011562 & 90 \\
\hline Micrococcus luteus & Micrococcus luteus strain RKA3 & EF463057.1 & 89 \\
\hline Staphylococcus aureus & Streptococcus agalactiae & AFO15927.1 & 99 \\
\hline Streptococcus pyogenes & Streptococcus uberis & U41048.1 & 98 \\
\hline Bacillus subtilis & Bacillus subtilis strain b17a & JX406823.1 & 92 \\
\hline Pseudomonas aeruginosa & Pseudomonas aeruginosa strain DSM 50071 & NR026078.1 & 98 \\
\hline Proteus vulgaris & Proteus vulgaris strain P.vul.Hk.3 & KX941831.1 & 96 \\
\hline Serratia marcescens & Serratia marcescens strain C7S3A & MF375920.1 & 96 \\
\hline \multicolumn{4}{|l|}{ Yeast } \\
\hline Pichia kluyveri & Pichia bruneiensis strain CLIB 1453 & LN909488.1 & 96 \\
\hline Pichia anomala & Pichia fermentans strain YM26567 & KY463385.1 & 98 \\
\hline Zygosaccharomyces rouxii & Zygosaccharomyces rouxii culture CBS & MF536908.1 & 94 \\
\hline Zygosaccharomyces bailii & Zygosaccharomyces bailii culture CBS:1170 & KY110238.1 & 99 \\
\hline Candida krusei & Candida inconspicua strain $1.626 \mathrm{~S}$ & JN417609.1 & 99 \\
\hline Torulopsis fragaria & Candida multigemmis culture CBS:6524 & KY106588.1 & 98 \\
\hline Geotrichum candidum & Geotrichum candidum SFC10 & MF186030.1 & 98 \\
\hline
\end{tabular}

${ }^{a}$ Microorganisms along the column were isolated from fruits

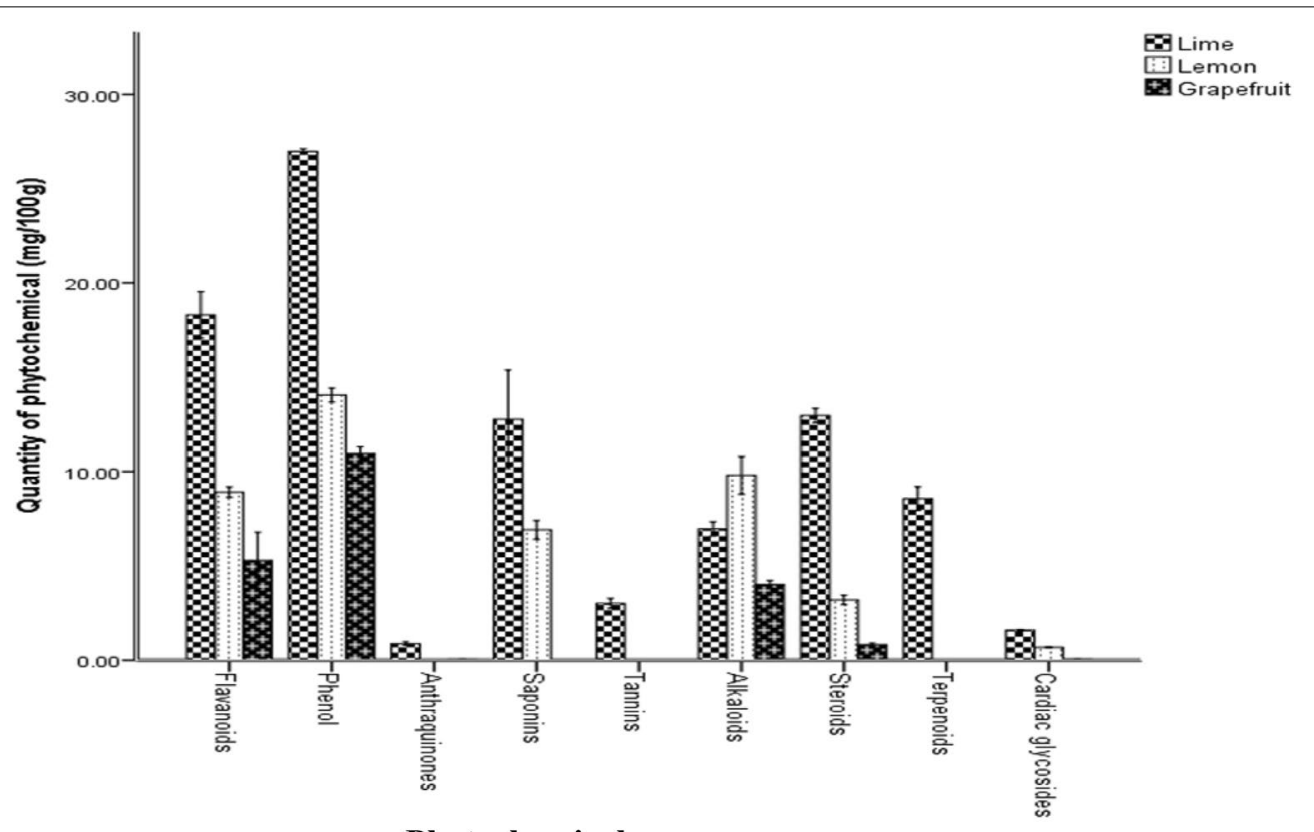

Phytochemicals

Fig. 1 Quantitative constituents (mg/100 g) of phytochemicals in Citrus essential oils 


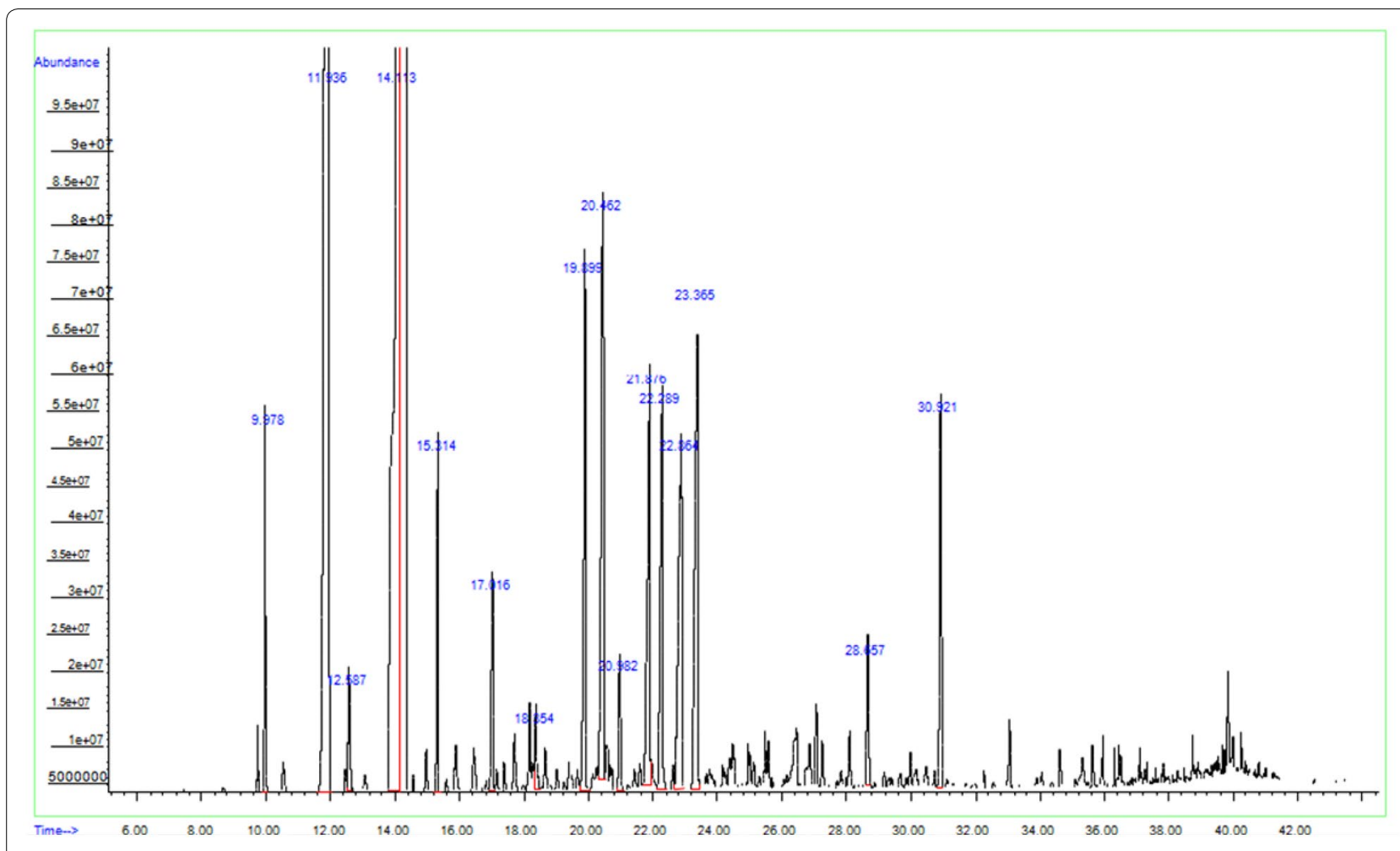

Fig. 2 Gas chromatogram of lime EO with peaks for various bioactive constituents

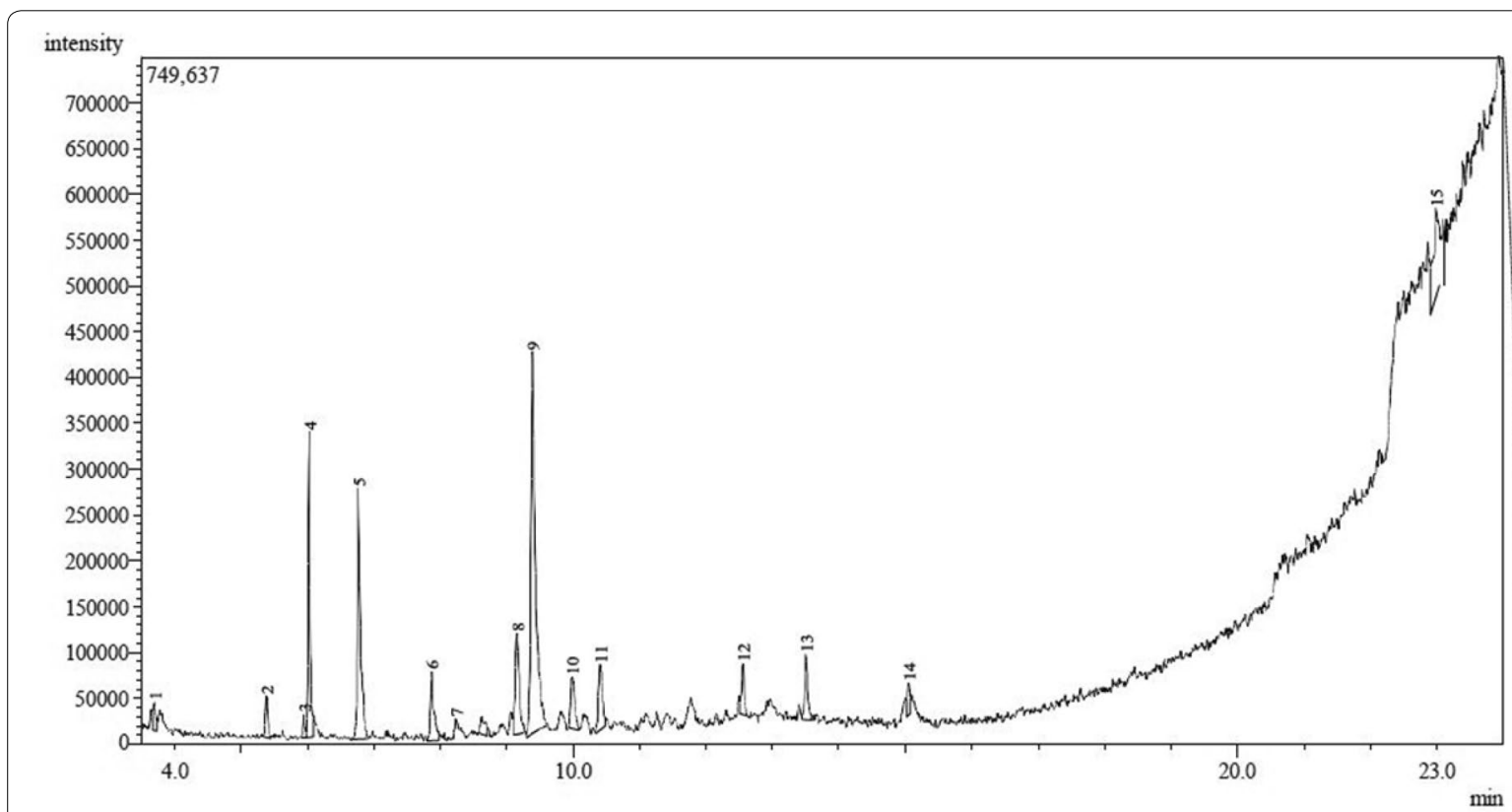

TIC $^{*} 1.00$

Fig. 3 Gas chromatogram of lemon EO with peaks for various bioactive constituents 
Table 4 Main bioactive component and the relative contents of CEOs using GC-MS

\begin{tabular}{|c|c|c|c|c|c|}
\hline & R. Time (min) & Peak area (\%) & Name of compounds & Molecular formula & $\begin{array}{l}\text { Molecular } \\
\text { weight (g/ } \\
\text { mol) }\end{array}$ \\
\hline \multicolumn{6}{|c|}{ Lime oil } \\
\hline 1 & 9.97 & 2.01 & Alpha-pinene & $\mathrm{C}_{10} \mathrm{H}_{16}$ & 136.24 \\
\hline 2 & 11.93 & 25.24 & Beta-pinene & $\mathrm{C}_{10} \mathrm{H}_{16}$ & 136.24 \\
\hline 3 & 12.58 & 0.97 & Beta-myrcene & $\mathrm{C}_{10} \mathrm{H}_{16}$ & 136.24 \\
\hline 4 & 14.12 & 27.86 & D-Limonene & $\mathrm{C}_{10} \mathrm{H}_{16}$ & 136.24 \\
\hline 5 & 15.31 & 2.47 & Alpha-phellandrene & $\mathrm{C}_{10} \mathrm{H}_{16}$ & 136.24 \\
\hline 6 & 17.01 & 1.71 & 3-Carene & $\mathrm{C}_{10} \mathrm{H}_{16}$ & 136.24 \\
\hline 7 & 18.35 & 0.89 & Trans-7-Oxabicyclo[4.1.0]heptane & $\mathrm{C}_{10} \mathrm{H}_{16} \mathrm{O}$ & 152.25 \\
\hline 8 & 19.90 & 5.84 & Terpinen-4-ol & $\mathrm{C}_{10} \mathrm{H}_{18} \mathrm{O}$ & 154.25 \\
\hline 9 & 20.46 & 7.52 & Alpha-terpineol & $\mathrm{C}_{10} \mathrm{H}_{18} \mathrm{O}$ & 154.25 \\
\hline 10 & 20.98 & 1.00 & Decanal & $\mathrm{C}_{10} \mathrm{H}_{20} \mathrm{O}$ & 156.20 \\
\hline 11 & 21.87 & 5.11 & 2,6-Octadien-1-ol & $\mathrm{C}_{8} \mathrm{H}_{14} \mathrm{O}$ & 126.20 \\
\hline 12 & 22.29 & 4.11 & 2,6-Octadienal & $\mathrm{C}_{8} \mathrm{H}_{12} \mathrm{O}$ & 124.18 \\
\hline 13 & 22.86 & 4.73 & Geraniol & $\mathrm{C}_{10} \mathrm{H}_{18} \mathrm{O}$ & 154.25 \\
\hline 14 & 23.36 & 5.57 & 3,7-dimethyl-citral & $\mathrm{C}_{10} \mathrm{H}_{16} \mathrm{O}$ & 152.24 \\
\hline 15 & 28.66 & 1.09 & 1,3,6,10-Dodecatetraene & $\mathrm{C}_{15} \mathrm{H}_{24}$ & 204.35 \\
\hline 16 & 30.92 & 2.91 & Beta-bisabolene & $\mathrm{C}_{15} \mathrm{H}_{24}$ & 204.35 \\
\hline \multicolumn{6}{|c|}{ Lemon oil } \\
\hline 1 & 3.69 & 1.44 & Isobutyl methyl ketone & $\mathrm{C}_{6} \mathrm{H}_{12} \mathrm{O}$ & 100.00 \\
\hline 2 & 5.37 & 1.65 & Alpha-phellandrene & $\mathrm{C}_{10} \mathrm{H}_{16}$ & 136.00 \\
\hline 3 & 5.93 & 0.88 & Sabinene & $\mathrm{C}_{10} \mathrm{H}_{16}$ & 136.00 \\
\hline 4 & 6.02 & 12.37 & Beta-pinene & $\mathrm{C}_{10} \mathrm{H}_{16}$ & 136.23 \\
\hline 5 & 6.76 & 14.40 & L-Limonene & $\mathrm{C}_{10} \mathrm{H}_{16}$ & 136.00 \\
\hline 6 & 7.86 & 4.56 & 3,7-dimethyl-1,3,6-Octatriene & $\mathrm{C}_{10} \mathrm{H}_{18} \mathrm{O}$ & 154.00 \\
\hline 7 & 8.62 & 1.50 & Fenchol & $\mathrm{C}_{10} \mathrm{H}_{18} \mathrm{O}$ & 154.25 \\
\hline 8 & 9.14 & 6.72 & Terpinen-4-ol & $\mathrm{C}_{10} \mathrm{H}_{18} \mathrm{O}$ & 154.00 \\
\hline 9 & 9.38 & 31.72 & Alpha-terpineol & $\mathrm{C}_{10} \mathrm{H}_{18} \mathrm{O}$ & 154.25 \\
\hline 10 & 9.97 & 3.39 & Neral & $\mathrm{C}_{10} \mathrm{H}_{16} \mathrm{O}$ & 152.00 \\
\hline 11 & 10.39 & 4.77 & Geranial & $\mathrm{C}_{10} \mathrm{H}_{16} \mathrm{O}$ & 152.00 \\
\hline 12 & 12.54 & 2.27 & Bergamotene & $\mathrm{C}_{15} \mathrm{H}_{24}$ & 204.00 \\
\hline 13 & 13.49 & 3.69 & Camphene & $\mathrm{C}_{10} \mathrm{H}_{16}$ & 136.00 \\
\hline 14 & 15.04 & 1.45 & Phthalic acid & $\mathrm{C}_{10} \mathrm{H}_{10} \mathrm{O}_{4}$ & 194.00 \\
\hline 15 & 22.97 & 9.18 & Cyclohexanol & $\mathrm{C}_{10} \mathrm{H}_{20} \mathrm{O}$ & 156.00 \\
\hline
\end{tabular}

\section{Inhibitory potential of CEOs against isolated microorganisms}

Table $5 \mathrm{a}-\mathrm{c}$ shows the zones of inhibition of CEOs and their combinations against the isolated microorganisms from fruits. The highest antibacterial activity of the CEOs was obtained with lime and lemon combination against Bacillus subtilis with inhibition zones of $26.8 \mathrm{~mm}$. The lowest MIC $(6.25 \mathrm{mg} / \mathrm{ml})$ was recorded on lime and lemon EOs synergy against Bacillus subtilis. Fusarium oxysporum was resistant to all the CEOs with no inhibition zone when tested against all the CEOs and their various combinations. Generally, lime and lemon oil synergy proved to be the most effective CEOs against the tested isolates. However, lime EO had the best antimicrobial activity when the extracts were used singly. The growth of Torulopsis fragaria was not inhibited by individual CEOs but was effective against the yeast at $100 \mathrm{mg} / \mathrm{ml}$ when the CEOs were combined. The comparative MIC, MBC and MFC of the CEOs on the isolated microorganisms are presented in Tables 6 and 7. The MIC results revealed that the CEOs inhibited the growth of most of the tested isolates between 12.5 and $100 \mathrm{mg} / \mathrm{ml}$.

\section{Discussion}

The findings of this study revealed an array of microorganisms isolated from spoilt pawpaw, apple, pineapple, watermelon, orange, cucumber, banana, tomato and 
Table 5 Zones of inhibition of CEOs at $100 \mathrm{mg} / \mathrm{ml}$ against some microorganisms isolated from spoilt fruits

\begin{tabular}{|c|c|c|c|c|c|c|c|c|}
\hline & A & B & C & $A+B$ & $A+C$ & $B+C$ & $A+B+C$ & Nystatin \\
\hline \multicolumn{9}{|l|}{ (a) Yeasts } \\
\hline Saccharomyces cerevisiae & $8.9 \pm 0.3$ & $9.4 \pm 0.1$ & $7.3 \pm 0.3$ & $12.5 \pm 0.5$ & $9.3 \pm 0.4$ & $7.9 \pm 0.2$ & $12.8 \pm 0.3$ & $14.6 \pm 0.5$ \\
\hline Candida tropicalis & $10.4 \pm 0.5$ & $9.8 \pm 0.2$ & $7.5 \pm 0.5$ & $16.5 \pm 0.5$ & $11.7 \pm 0.5$ & $11.3 \pm 0.6$ & $14.7 \pm 0.5$ & $16.6 \pm 0.6$ \\
\hline Candida krusei & $13.3 \pm 0.2$ & $13.6 \pm 0.3$ & $13.3 \pm 0.5$ & $16.3 \pm 0.3$ & $12.8 \pm 0.2$ & $13.7 \pm 0.6$ & $16.7 \pm 0.5$ & $18.6 \pm 0.5$ \\
\hline Kluyveromyces marxianus & $12.3 \pm 0.5$ & $12.3 \pm 0.5$ & $12.1 \pm 0.2$ & $13.0 \pm 1.0$ & $13.2 \pm 0.3$ & $12.8 \pm 0.3$ & $13.7 \pm 0.2$ & $20.0 \pm 1.0$ \\
\hline Torulopsis fragaria & 0.0 & 0.0 & 0.0 & $7.8 \pm 0.3$ & $7.5 \pm 0.5$ & $7.3 \pm 0.3$ & $7.6 \pm 0.5$ & $16.7 \pm 0.5$ \\
\hline Pichia kluyveri & $17.6 \pm 0.5$ & $15.3 \pm 0.5$ & $13.3 \pm 0.5$ & $18.3 \pm 0.5$ & $16.3 \pm 0.2$ & $15.8 \pm 0.3$ & $16.5 \pm 0.5$ & $17.7 \pm 0.5$ \\
\hline Pichia fermentans & $11.7 \pm 0.6$ & $12.7 \pm 0.6$ & $10.8 \pm 0.2$ & $15.0 \pm 1.0$ & $14.3 \pm 0.5$ & $14.2 \pm 0.5$ & $15.2 \pm 0.2$ & $18.3 \pm 0.6$ \\
\hline Pichia anomala & $15.1 \pm 0.1$ & $12.3 \pm 0.5$ & $10.6 \pm 0.3$ & $15.7 \pm 0.6$ & $14.5 \pm 0.5$ & $13.3 \pm 0.5$ & $14.5 \pm 0.8$ & $19.7 \pm 0.5$ \\
\hline Geotrichum candidum & $9.7 \pm 0.6$ & $10.7 \pm 0.2$ & $8.3 \pm 0.5$ & $12.0 \pm 0.0$ & $9.5 \pm 0.8$ & $10.3 \pm 0.5$ & $12.7 \pm 0.5$ & $18.0 \pm 0.0$ \\
\hline Zygosaccharomyces rouxii & $9.4 \pm 0.5$ & $7.2 \pm 0.3$ & $5.8 \pm 0.2$ & $10.3 \pm 0.6$ & $10.0 \pm 0.0$ & $8.3 \pm 0.5$ & $11.9 \pm 0.1$ & $14.7 \pm 0.5$ \\
\hline Zygosaccharomyces bailii & $7.4 \pm 0.5$ & $7.9 \pm 0.5$ & $6.3 \pm 0.2$ & $8.0 \pm 0.0$ & $7.3 \pm 0.5$ & $9.3 \pm 0.6$ & $8.7 \pm 0.4$ & $13.7 \pm 0.5$ \\
\hline \multicolumn{9}{|l|}{ (b) Mould } \\
\hline Aspergillus niger & $9.3 \pm 0.5$ & $8.3 \pm 0.5$ & $4.3 \pm 0.5$ & $15.7 \pm 0.3$ & $9.7 \pm 0.5$ & $6.7 \pm 0.3$ & $12.1 \pm 0.29$ & $15.3 \pm 0.2$ \\
\hline Aspergillus flavus & $8.1 \pm 0.3$ & $7.8 \pm 1.0$ & $5.5 \pm 0.8$ & $13.3 \pm 0.7$ & $5.2 \pm 0.5$ & $5.0 \pm 0.5$ & $11.7 \pm 0.58$ & $16.5 \pm 0.5$ \\
\hline Aspergillus fumigatus & $9.0 \pm 1.0$ & $8.0 \pm 1.0$ & $3.7 \pm 0.5$ & $14.8 \pm 0.3$ & $10.3 \pm 0.6$ & $7.2 \pm 0.2$ & $11.8 \pm 0.76$ & $17.3 \pm 0.5$ \\
\hline Aspergillus parasiticus & $7.8 \pm 0.2$ & $8.2 \pm 0.7$ & $7.3 \pm 0.6$ & $9.5 \pm 0.5$ & $9.3 \pm 0.3$ & $9.1 \pm 0.7$ & $9.1 \pm 0.29$ & $19.1 \pm 0.7$ \\
\hline Mucor piriformis & $17.5 \pm 0.0$ & $17.7 \pm 0.5$ & $16.3 \pm 0.5$ & $22.8 \pm 0.2$ & $20.8 \pm 0.3$ & $19.6 \pm 0.5$ & $20.5 \pm 0.50$ & $18.6 \pm 0.5$ \\
\hline Mucor racemosis & $14.6 \pm 0.5$ & $13.3 \pm 0.3$ & $15.3 \pm 0.5$ & $13.8 \pm 0.2$ & $15.7 \pm 0.7$ & $15.0 \pm 0.5$ & $16.0 \pm 1.00$ & $18.5 \pm 0.5$ \\
\hline Alternaria alternata & $16.5 \pm 0.5$ & $14.7 \pm 0.5$ & $15.0 \pm 1.0$ & $16.7 \pm 0.5$ & $15.6 \pm 0.5$ & $15.1 \pm 0.3$ & $17.7 \pm 0.58$ & $20.3 \pm 0.5$ \\
\hline Fusarium avenaceum & $6.1 \pm 0.2$ & $5.3 \pm 0.5$ & 0.0 & $8.5 \pm 0.5$ & $6.3 \pm 0.5$ & 0.0 & $8.3 \pm 0.29$ & $15.3 \pm 0.5$ \\
\hline Fusarium oxysporum & 0.0 & 0.0 & 0.0 & 0.0 & 0.0 & 0.0 & 0.0 & $19.3 \pm 0.5$ \\
\hline Fusarium solani & $4.6 \pm 0.5$ & $5.7 \pm 0.5$ & $4.2 \pm 0.3$ & $9.5 \pm 0.5$ & $8.7 \pm 0.5$ & $8.3 \pm 0.5$ & $10.7 \pm 0.5$ & $21.6 \pm 0.5$ \\
\hline Penicillium digitatum & $21.3 \pm 0.5$ & $21.0 \pm 1.0$ & $17.3 \pm 0.6$ & $23.7 \pm 0.5$ & $18.6 \pm 0.5$ & $18.0 \pm 0.5$ & $23.2 \pm 0.2$ & $17.6 \pm 0.5$ \\
\hline Penicillium expansum & $18.9 \pm 0.5$ & $18.7 \pm 0.5$ & $17.3 \pm 0.6$ & $19.7 \pm 0.2$ & $18.8 \pm 0.7$ & $18.3 \pm 0.2$ & $20.7 \pm 0.5$ & $16.5 \pm 0.8$ \\
\hline Rhizopus oryzae & $11.7 \pm 0.5$ & $12.5 \pm 0.5$ & $6.8 \pm 0.7$ & $14.7 \pm 0.5$ & $9.8 \pm 0.7$ & $9.8 \pm 0.2$ & $14.3 \pm 0.5$ & $19.3 \pm 0.5$ \\
\hline Rhizopus stolonifer & $12.3 \pm 0.5$ & $11.3 \pm 0.5$ & $8.5 \pm 0.5$ & $15.0 \pm 1.0$ & $9.5 \pm 0.5$ & $9.8 \pm 0.3$ & $15.3 \pm 0.5$ & $18.7 \pm 0.5$ \\
\hline \multicolumn{9}{|l|}{ (c) Bacteria } \\
\hline Bacillus cereus & $14.6 \pm 0.3$ & $14.6 \pm 0.5$ & $7.6 \pm 0.5$ & $17.1 \pm 0.2$ & $15.3 \pm 0.5$ & $15.1 \pm 0.2$ & $16.7 \pm 0.5$ & $19.1 \pm 0.1$ \\
\hline Bacillus subtilis & $18.2 \pm 0.2$ & $17.5 \pm 0.5$ & $16.3 \pm 0.5$ & $26.8 \pm 0.2$ & $20.3 \pm 0.5$ & $19.3 \pm 0.5$ & $24.3 \pm 0.6$ & $20.3 \pm 0.5$ \\
\hline Micrococcus /uteus & $15.3 \pm 0.5$ & $13.6 \pm 0.5$ & $10.6 \pm 0.5$ & $14.3 \pm 0.5$ & $12.6 \pm 0.5$ & $12.3 \pm 0.2$ & $16.7 \pm 0.5$ & $18.3 \pm 0.5$ \\
\hline Serratia marcescens & $8.7 \pm 0.5$ & $8.3 \pm 0.5$ & $7.6 \pm 0.5$ & $11.6 \pm 0.5$ & $11.3 \pm 0.5$ & $11.3 \pm 0.5$ & $12.3 \pm 0.8$ & $17.3 \pm 0.5$ \\
\hline Lactobacillus plantarum & $6.6 \pm 0.5$ & $4.3 \pm 0.5$ & $3.3 \pm 0.5$ & $9.8 \pm 0.7$ & $5.3 \pm 0.5$ & $5.3 \pm 0.5$ & $8.3 \pm 0.5$ & $17.3 \pm 0.2$ \\
\hline Streptococcus uberis & $17.1 \pm 0.2$ & $16.1 \pm 0.2$ & $13.1 \pm 0.3$ & $16.8 \pm 0.5$ & $15.5 \pm 0.5$ & $13.7 \pm 0.5$ & $16.7 \pm 0.5$ & $19.6 \pm 0.5$ \\
\hline Proteus vulgaris & $16.1 \pm 0.2$ & $16.5 \pm 0.1$ & $16.2 \pm 0.2$ & $16.8 \pm 0.2$ & $16.3 \pm 0.5$ & $15.1 \pm 0.2$ & $19.3 \pm 0.5$ & $18.5 \pm 0.5$ \\
\hline Streptococcus agalactiae & $18.8 \pm 0.5$ & $15.6 \pm 0.5$ & $14.5 \pm 0.5$ & $17.8 \pm 0.7$ & $17.6 \pm 0.2$ & $17.1 \pm 0.2$ & $19.1 \pm 0.2$ & $16.6 \pm 0.5$ \\
\hline Pseudomonas aeruginosa & $14.6 \pm 0.2$ & $15.5 \pm 0.5$ & $11.6 \pm 0.2$ & $16.8 \pm 0.2$ & $14.4 \pm 0.5$ & $15.4 \pm 0.2$ & $16.0 \pm 0.0$ & $15.7 \pm 0.8$ \\
\hline Erwinia cacticida & $11.3 \pm 0.6$ & $11.3 \pm 0.5$ & $9.0 \pm 1.0$ & $12.1 \pm 0.2$ & $11.5 \pm 0.5$ & $11.6 \pm 0.2$ & $11.8 \pm 0.2$ & $18.3 \pm 0.5$ \\
\hline
\end{tabular}

A-lime essential oil, B-lemon essential oil, C—grapefruit essential oil

African star apple. The predominance of fungi in the fruit samples is in agreement with the findings of Singh and Sharma [23] who reported that spoilage of fruits is mainly caused by fungi. High sugar concentrations in fruits assist the development of fungi because they are xerophilic in nature [24]. The presence of pathogens on susceptible host fruit, availability of various essential nutrients such as sugars, minerals, vitamins, amino acids as well as suitable environmental conditions supports fungi colonization and disease expression [25]. The variation in occurrence of spoilage microorganisms of fruits can be related to type of fruits, farm location, method of harvest and level of hygiene maintained by fruit sellers/handlers.

Some of the microorganisms isolated from fruit were Penicillium spp., Rhizopus spp., Fusarium oxysporum and Mucor piriformis. Akintobi et al. [26] and Tafinta et al. [27] have isolated similar microorganisms from 
Table 6 Comparative minimum inhibitory concentrations $(\mathrm{mg} / \mathrm{ml})$ of CEOs against bacterial and fungal isolates

\begin{tabular}{|c|c|c|c|c|c|c|c|}
\hline Isolates & $A$ & B & $\mathrm{C}$ & $A+B$ & $A+C$ & $B+C$ & $A+B+C$ \\
\hline Saccharomyces cerevisiae & 75 & 75 & 100 & 50 & 75 & 100 & 50 \\
\hline Candida tropicalis & 75 & 75 & 100 & 25 & 75 & 75 & 50 \\
\hline Candida krusei & 50 & 50 & 50 & 25 & 50 & 50 & 25 \\
\hline Kluyveromyces marxianus & 50 & 50 & 50 & 50 & 50 & 50 & 50 \\
\hline Torulopsis fragaria & 0.0 & 0.0 & 0.0 & 100 & 100 & 100 & 100 \\
\hline Pichia kluyveri & 12.5 & 25 & 50 & 12.5 & 25 & 25 & 25 \\
\hline Pichia fermentans & 25 & 25 & 25 & 12.5 & 12.5 & 12.5 & 12.5 \\
\hline Pichia anomala & 12.5 & 12.5 & 25 & 12.5 & 12.5 & 12.5 & 12.5 \\
\hline Geotrichum candidum & 25 & 25 & 50 & 25 & 50 & 50 & 25 \\
\hline Zygosaccharomyces rouxii & 75 & 100 & 100 & 75 & 75 & 100 & 75 \\
\hline Zygosaccharomyces bailii & 100 & 100 & 100 & 100 & 100 & 100 & 100 \\
\hline Aspergillus niger & 50 & 75 & 100 & 25 & 75 & 75 & 50 \\
\hline Aspergillus flavus & 50 & 75 & 100 & 50 & 100 & 100 & 50 \\
\hline Aspergillus fumigatus & 75 & 75 & 100 & 25 & 75 & 75 & 50 \\
\hline Aspergillus parasiticus & 75 & 75 & 75 & 75 & 75 & 75 & 50 \\
\hline Mucor piriformis & 12.5 & 25 & 12.5 & 25 & 12.5 & 12.5 & 12.5 \\
\hline Mucor racemosis & 25 & 50 & 25 & 50 & 25 & 25 & 25 \\
\hline Fusarium oxysporum & 0.0 & 0.0 & 0.0 & 0.0 & 0.0 & 0.0 & 0.0 \\
\hline Fusarium avenaceum & 100 & 100 & 0.0 & 75 & 100 & 0.0 & 75 \\
\hline Fusarium solani & 100 & 100 & 100 & 50 & 50 & 50 & 25 \\
\hline Penicillium digitatum & 12.5 & 12.5 & 25 & 12.5 & 25 & 25 & 12.5 \\
\hline Penicillium expansum & 25 & 25 & 25 & 25 & 25 & 25 & 12.5 \\
\hline Rhizopus oryzae & 75 & 75 & 100 & 50 & 100 & 100 & 50 \\
\hline Rhizopus stolonifer & 75 & 75 & 100 & 50 & 100 & 100 & 50 \\
\hline Alternaria alternata & 25 & 50 & 50 & 25 & 50 & 25 & 25 \\
\hline Lactobacillus plantarum & 75 & 100 & 100 & 75 & 100 & 100 & 75 \\
\hline Bacillus cereus & 25 & 25 & 50 & 25 & 25 & 25 & 25 \\
\hline Micrococcus luteus & 25 & 50 & 50 & 50 & 50 & 50 & 50 \\
\hline Streptococcus uberis & 25 & 25 & 50 & 25 & 25 & 25 & 25 \\
\hline Bacillus subtilis & 12.5 & 12.5 & 25 & 6.25 & 12.5 & 12.5 & 12.5 \\
\hline Proteus vulgaris & 25 & 25 & 25 & 25 & 25 & 25 & 12.5 \\
\hline Serratia marcescens & 50 & 50 & 50 & 25 & 25 & 25 & 25 \\
\hline Streptococcus agalactiae & 12.5 & 25 & 25 & 25 & 25 & 25 & 12.5 \\
\hline Pseudomonas aeruginosa & 12.5 & 12.5 & 50 & 12.5 & 25 & 25 & 25 \\
\hline Erwinia cacticida & 25 & 25 & 75 & 25 & 50 & 50 & 25 \\
\hline
\end{tabular}

A—lime oil, B—lemon oil, C—grapefruit oil, 0.0 -no inhibition detected when $\geq 100 \mathrm{mg} / \mathrm{ml}$ was used

pawpaw, orange and tomato in Ibadan, Nigeria. In a similar context, deterioration of fruits (pawpaw, orange, tomato, pineapple and watermelon) had reportedly linked to Aspergillus niger, Aspergillus flavus and Rhizopus stolonifer by Chukwuka et al. [28] and Mailafia et al. [25]. These microorganisms caused different types of spoilage in fruits, which had been associated with economic losses. Soft rot of apple caused by blue mould; Penicillium spp. is the most concerning postharvest disease of apple worldwide [29], while species of Aspergillus, Rhizopus and Mucor are responsible for the soft rots of orange [30]. The fungi invade the apple tissues by releasing pectic enzymes that break down the gel-like pectic compounds that cement the apple cells together [31]. Penicillium digitatum causes a devastating and rapid green rot of Citrus spp., while blue rot of apples and pears is caused by Penicillium expansum [32]. Rhizopus stolonifer and Mucor piriformis are reportedly responsible for the rapid decay of soft fruits such as raspberries, tomatoes, orange and loganberries. These moulds can spread rapidly especially on commodities stored at temperatures above $20^{\circ} \mathrm{C}$ [33]. $M$. 
Table 7 Comparative minimum bactericidal and fungicidal concentrations $(\mathrm{mg} / \mathrm{ml})$ of CEOs against microbial isolates

\begin{tabular}{|c|c|c|c|c|c|c|c|}
\hline Isolates & A & B & C & $A+B$ & $A+C$ & $B+C$ & $A+B+C$ \\
\hline Saccharomyces cerevisiae & 75 & 100 & $>100$ & 75 & 100 & $>100$ & 75 \\
\hline Candida tropicalis & 75 & 100 & $>100$ & 50 & 100 & 100 & 75 \\
\hline Candida krusei & 50 & 25 & 50 & 25 & 50 & 50 & 25 \\
\hline Kluyveromyces marxianus & 75 & 75 & 100 & 75 & 75 & 75 & 75 \\
\hline Torulopsis fragaria & 0.0 & 0.0 & 0.0 & 0.0 & 0.0 & 0.0 & $>100$ \\
\hline Pichia kluyveri & 25 & 50 & 75 & 25 & 25 & 50 & 25 \\
\hline Pichia fermentans & 50 & 50 & 50 & 25 & 25 & 25 & 25 \\
\hline Pichia anomala & 50 & 50 & 50 & 25 & 25 & 25 & 25 \\
\hline Geotrichum candidum & 50 & 50 & 75 & 25 & 75 & 75 & 50 \\
\hline Zygosaccharomyces rouxii & $>100$ & $>100$ & $>100$ & $>100$ & $>100$ & $>100$ & $>100$ \\
\hline Zygosaccharomyces bailii & $>100$ & $>100$ & $>100$ & $>100$ & $>100$ & $>100$ & $>100$ \\
\hline Aspergillus niger & 100 & 100 & $>100$ & 50 & 75 & 100 & 50 \\
\hline Aspergillus flavus & $>100$ & $>100$ & $>100$ & $>100$ & $>100$ & $>100$ & $>100$ \\
\hline Aspergillus fumigatus & 100 & 100 & $>100$ & 50 & 75 & 75 & 50 \\
\hline Aspergillus parasiticus & 100 & 100 & 100 & 100 & 100 & 100 & 100 \\
\hline Mucor piriformis & 50 & 75 & 50 & 75 & 50 & 50 & 50 \\
\hline Mucor racemosis & 50 & 75 & 50 & 75 & 50 & 75 & 50 \\
\hline Fusarium oxysporum & 0.0 & 0.0 & 0.0 & 0.0 & 0.0 & 0.0 & 0.0 \\
\hline Fusarium avenaceum & $>100$ & $>100$ & 0.0 & $>100$ & $>100$ & 0.0 & $>100$ \\
\hline Fusarium solani & $>100$ & $>100$ & $>100$ & 100 & 100 & 100 & 75 \\
\hline Penicillium digitatum & 25 & 25 & 50 & 12.5 & 25 & 25 & 12.5 \\
\hline Penicillium expansum & 25 & 25 & 25 & 25 & 25 & 25 & 25 \\
\hline Rhizopus oryzae & 100 & 100 & $>100$ & 75 & $>100$ & $>100$ & 75 \\
\hline Rhizopus stolonifer & 100 & 100 & $>100$ & 75 & $>100$ & $>100$ & 75 \\
\hline Alternaria alternata & 75 & 50 & 50 & 75 & 75 & 75 & 50 \\
\hline Bacillus cereus & 25 & 25 & 50 & 25 & 25 & 25 & 25 \\
\hline Micrococcus /uteus & 75 & 50 & 75 & 50 & 75 & 75 & 50 \\
\hline Streptococcus uberis & 50 & 50 & 100 & 25 & 50 & 50 & 25 \\
\hline Bacillus subtilis & 25 & 25 & 50 & 25 & 25 & 25 & 25 \\
\hline Proteus vulgaris & 50 & 50 & 50 & 50 & 50 & 50 & 25 \\
\hline Serratia marcescens & 100 & 100 & 100 & 50 & 50 & 50 & 50 \\
\hline Streptococcus agalactiae & 25 & 50 & 50 & 50 & 50 & 50 & 25 \\
\hline Pseudomonas aeruginosa & 12.5 & 12.5 & 50 & 12.5 & 25 & 25 & 25 \\
\hline Erwinia cacticida & 25 & 25 & 75 & 25 & 50 & 50 & 25 \\
\hline
\end{tabular}

A-lime oil, B-lemon oil, C—grapefruit oil, 0.0—no inhibition detected when $\geq 100 \mathrm{mg} / \mathrm{ml}$ was used

piriformis has been described as a destructive pathogen of strawberries [30]. Aspergillus niger, one of the fungi isolated, is known to be responsible for black mould rot of fruits such as cherry, grapes, banana and mango in the report of Sharma [34]. Akintobi et al. [26] who reported that species of Rhizopus, Aspergillus, Penicillium and Candida tropicalis are responsible for rot of pawpaw. The isolation of Aspergillus niger, Rhizopus stolonifer, Mucor species from tomato agreed with studies of Chuku et al. [35] and Akinmusire [36] who reported that $A$. flavus and $A$. fumigatus were major spoilage microorganisms of tomato. Fungal isolates:
Aspergillus, Mucor, Rhizopus, Penicillium and Fusarium have been reported to be the major deteriorative agents of watermelon and pineapple [37].

The presence of these microorganisms on fruits from the farm to the final products can have a health implication on human. Out of the fungi isolated, the presence of Aspergillus niger, which causes a black mould in certain fruits and vegetables as well as producing potent mycotoxins like aflatoxin and ochratoxin agreed with the work of Akinmusire [36] and Onuorah and Orji [38]. Poostforoushfard et al. [39] reported the contamination of apple juices in Southern Iran by patulin that was 
produced by $P$. expansum. This mycotoxin is often present in apple juice and apple products; although ochratoxin $\mathrm{A}$ is predominantly a problem in commodities such as cereals, cocoa and coffee, it is also found in grapes and grape products. Therefore, the biological control of fruits spoilage is quite important to guarantee the safety of the consumers.

Some plants possess antimicrobial activities because they contain secondary metabolites that are responsible for bioactivity to prevent colonization of spoilage microorganisms. In this study, the antimicrobial activities of CEOs were tested against isolated microorganisms from fruits. The presence of phytochemicals in CEOs confirmed the claim of Mathew et al. [40] and Ozcan et al. [41]. The researchers stated that CEOs possess abundant phytochemicals. These phytochemicals varied in Citrus spp., as lime EO was found to have more phytochemicals than lemon and grapefruit EOs. The findings agreed in part to the results of Oikeh et al. [11] who revealed the presence of alkaloids, phenols, flavonoids, steroids, terpenoids, saponins and cardiac glycosides in lime, lemon and grape juice. However, they did not detect the presence of anthraquinones and tannins in lime, while phenols and flavonoids were detected in low amount in lime and lemon. These variations may be influenced by various factors such as genotypic differences, geographic and climatic conditions, time of harvest, age of the Citrus spp., extraction techniques adopted and even cultural practices [42].

Apart from the antimicrobial efficacy of these phytochemicals, they have been reported to be of higher medicinal importance. Saponin, which was present in lime and lemon EOs, is reported to boost the immune system and fight against pathogens that cause infections [43]. Flavonoid was abundantly present in the CEOs. It is water-soluble polyphenolic molecules that are mainly responsible for the colouring of fruits and vegetables [44]. Flavonoids have been the major antioxidant compounds in fruits and vegetables, hence it promotes health benefits such as anti-cancer, diuretics, antispasmodic, antidiabetic and anti-viral $[45,46]$. These may make the EOs of C. aurantifolia, C. limon and C. paradisi useful to prevent some diseases.

The CEOs possessed antimicrobial activities against fungi and bacteria. The highest antifungal activity of CEOs tested in this study was in agreement with previous findings of Tullio et al. [47]. Although, a comparison of studies with different methodologies is difficult, our findings correlate with that of Vitoratos et al. [5] who showed that lemon EO was effective against Penicillium spp. and Botrytis cinerea. The efficient fungal growth reduction by oil of $C$. aurantifolia in this work correlates with the findings of Van et al. [48] who reported the growth reduction in Fusarium species by the CEO. Growth inhibition of Bacillus cereus with the application of CEOs was reported by Madhuri et al. [49]. The inhibitory potential of CEOs against tested microorganisms could be associated with the presence of monoterpenes. The main constituents of the lime oil of this study are limonene, beta-pinene and alpha-terpineol, which are classified as monoterpenes [50]. This agreed with the report of Jafari et al. [51] who pointed monoterpene hydrocarbons as the most abundant compounds in $\mathrm{EO}$ of lime. Limonene in studied lime EO corroborates the study of Ammad et al. [52] who reported that limonene was the major bioactive compound isolated from Citrus and has strong antifungal activity against fungi that caused decay on grapevine wood. The presence of limonene in lemon EO in this work agreed with the findings of Ammad et al. [52] who detected $70 \%$ limonene in lemon $\mathrm{EO}$, although the quantity obtained in this work was lower than theirs. This variation in composition could be a function of factors such as developmental stage of the plants, the organs harvested, the period and the geographical area of harvest. The antimicrobial efficacy of limonene against microorganisms could be its ability to interfere in energy homeostasis and caused cell membrane damage [53]. Studies carried out by Budzyńska et al. [54] and Mondello et al. [55] reported the strong antibacterial activity of terpinen4-ol, which was found to be present in both lime and lemon.

Lime EO had the best antimicrobial activity when the extracts were used singly, followed by lemon $\mathrm{EO}$ and least by grapefruit EO. The presence of more phytochemicals in lime EO could be responsible for better antimicrobial efficiency. EOs containing high composition of aldehyde or phenol were reported to exhibit a high inhibitory effect, followed by those containing alcohol, ketone and ether $[47,56]$. Research by Ozcan et al. [41] also reported the health benefits of phenols from fruits of Citrus especially, their antioxidant and antimicrobial properties. Phenols have anti-carcinogenic and anti-mutagenic effects since they act as protective agents of DNA against free radicals [41]. The high inhibitory potential of lime EO could be due to the presence of cyclic terpene hydrocarbons like alpha-pinene, beta-pinene and limonene that have showed a toxic effect on microorganisms [57]. The lipophilic character of these cyclic terpenes makes them to accumulate in the lipid structure of the cell wall, which then causes denaturing of proteins and loss of cell membrane integrity leading to cytoplasmic leakage and finally death of microorganisms [58]. Hence, it is not surprising that lime EO proved to be the best microbial growth inhibitor because of phenol as the major contributor to its bioactivity. However, lime and lemon EOs synergistically proved to be the most effective CEOs against 
the tested microorganisms. The better antimicrobial efficacy may be as a result of the mixture of chemically different biologically active compounds such as terpenes, both oxygenated and non-oxygenated [59]. Dinan et al. [60] revealed that the mode of action of EOs takes place against microorganisms by attacking the microbial cell wall, which resulted in increased permeability and loss of cellular constituents, acidification of the cytoplasmic content; blocking the production of cellular energy and synthesis of structural components; and, finally, destruction of genetic material leading to the death of the microorganisms [61]. The synergistic activity of CEOs may be due to their actions on microbial cell membrane.

The antimicrobial potency of CEOs may be influenced by the interactions between its constituents and is better in synergism than when its major components are tested separately [62]. Lambert et al. [63] revealed that the synergy between thyme oil's main constituents, carvacrol and thymol, facilitated its antibacterial efficacies against Staphylococcus aureus and Pseudomonas aeruginosa. Therefore, the synergism between different components of two or more CEOs plays an important role in proffering a better antimicrobial activity than when used in solitary. As a result, plant secondary compounds possess several modes of action on fungal strains, but a different antimicrobial mechanism of action of the CEOs to that of currently used antibiotics further emphasizes their value as antimicrobial agents against multi-drug-resistant strains. The evaluation of the antifungal activity of CEOs revealed their inhibitory effect against most of the fungal strains, but the MIC and MFC results indicated that higher concentrations inhibit fungus more efficiently than when diluted. In the findings of Sharma and Tripathi [64], a concentration of $3.0 \mathrm{mg} / \mathrm{ml}$ of EO from C. sinensis was potent enough to inhibit mycelial growth of postharvest spoilage pathogens and significantly reduced the growth of $A$. niger in a dose-response manner. The CEOs exhibited different degrees of inhibition on the growth of the tested fungi, but Penicillium digitatum was the most sensitive to CEOs inhibition. This agreed with findings of Caccioni et al. [65] and Jing et al. [66] who reported the antifungal efficacies of $\mathrm{EO}$ from $C$. sinensis against $P$. digitatum and P. italicum.

\section{Conclusion}

CEOs exhibited pronounced inhibitory potential against microorganisms associated with fruits spoilage. Hence, the availability of Citrus peels that are often considered as waste in developing countries can be exploited for medicinal uses. The bioactive ingredients in CEOs can therefore be used as alternative to chemical preservative agents that have displayed some side effects.

\section{Abbreviation}

CEOs: Citrus essential oils; EO: essential oil; MIC: minimum inhibitory concentration; MBC: minimum bactericidal concentration; MFC: minimum fungicidal concentration; GC-MS: gas chromatography-mass spectrometry; CLSI: Clinical and Laboratory Standards Institute.

\section{Acknowledgements}

The authors appreciate the technical support received from the technologists in Department of Microbiology, The Federal University of Technology, Akure, Nigeria.

\section{Authors' contributions \\ All the authors made substantial contributions to the conception, design and acquisition of the research study. OBA carried out the data collection, and OCO did the interpretation. The manuscript was drafted by OCO and OBA, revised by all authors to meet up with the intellectual content. All authors read and approved the final manuscript.}

\section{Funding}

Not applicable.

\section{Availability of data and materials}

All the data and materials are available for use.

\section{Ethics approval and consent to participate}

This manuscript is an original research and has not been published in other journals.

\section{Consent for publication}

All the authors agreed to publish the journal.

\section{Competing interests}

The authors declare that they have no competing interests.

\section{Author details}

${ }^{1}$ Department of Microbiology, The Federal University of Technology, P.M.B. 704, Akure, Nigeria. ${ }^{2}$ Biotechnology Unit, Department of Biological Sciences, Kings University, P.M.B. 555, Odeomu, Nigeria.

Received: 24 May 2019 Accepted: 19 July 2019

Published online: 19 November 2019

\section{References}

1. Nti CA, Hagan J, Bagina F, Seglah M. Knowledge of nutrition and health benefits and frequency of consumption of fruits and vegetables among Ghanaian homemakers. Afr J Food Sci. 2011;5(6):333-9.

2. Al-Hindi RR, Al-Najada AR, Mohammed SA. Isolation and identification of some fruit spoilage fungi. Afri J Microbiol Res. 2011;5(4):443-9.

3. Fox T, Fimeche C. Global food Waste Not, Want Not. England and Wales: IMechE; 2013.

4. Olayemi FF, Adegbola JA, Bamishaiye El, Awagu EF. Assessment of postharvest losses of some selected crops in eight local government areas of Rivers State, Nigeria. Asian J Rural Dev. 2012;2:13-23.

5. Vitoratos A, Bilalis D, Karkanis A, Efthimiadou A. Antifungal activity of plant essential oils against Botrytis cinerea, Penicillium italicum and Penicillium digitatum. Notulae Botanicae Horti Agrobotanici Cluj-Napoca. 2013;41(1):86-92

6. Thiyam B, Sharma GD. Isolation and identification of fungi associated with local fruits of Barak Valley. Curr World Environ. 2013;8(2):319-22.

7. Tripathi P, Dubey NK. Exploitation of natural products as an alternative strategy to control postharvest fungal rotting of fruit and vegetables. Postharvest Biol Technol. 2004;32:235-45.

8. Founou LL, Founou RC, Essack SY. Antibiotic resistance in the food chain: a developing country-perspective. Front Microbiol. 2016;7:1881

9. Gatto MA, Ippolito A, Linsalata V, Cascarano NA, Nigro F, Vanadia S, Di Venere D. Activity of extracts from wild edible herbs against postharvest fungal diseases of fruit and vegetables. Postharvest Biol Technol. $2011: 61: 72-82$ 
10. Oikeh El, Omoregie ES, Oviasogie FE, Oriakhi K. Phytochemical, antimicrobial, and antioxidant activities of different citrus juice concentrates. Food Sci Nutr. 2016;4(1):103-9.

11. Sanders ER. Aseptic laboratory techniques: plating methods. J Vis Exp. 2012;63:3064

12. Krieg NR, Staley JT, Brown, DR, Hedlund, BP, Paster BJ, Ward, NL, Ludwig W, Whitman WB. The Bacteroidetes, Spirochaetes, Tenericutes (Mollicutes), Acidobacteria, Fibrobacteres, Fusobacteria, Dictyoglomi, Gemmatimonadetes, Lentisphaerae, Verrucomicrobia, Chlamydiae, and Planctomycetes, 2nd ed., vol. 4. New York: Springer; 2010. https://doi.org/10.1007/978-0387-68572-4.

13. Domsch KH, Gams W, Anderson TH. Compedium of soil fungi. London: Academic Press; 1993.

14. Samson RA, Houbraken J, Thrane U, Frisvad JC, Andersen B. Fungi and indoor fungi. CBS laboratory manual series. Utrecht: CBS-KNAW Fungal Biodiversity Centre; 2010.

15. Barnett JA, Payne RW, Yarrow O. Yeast characteristics and identification. 3rd ed. London: Cambridge University Press; 2000.

16. Zolan ME, Pukkila PJ. Inheritance of DNA methylation in Coprinus cinereus. Mol Cell Biol. 1986:6:195-200.

17. Seema S, Veena $U$, Bhatt RP. Inhibitory effect of essential oils against Trichosporon ovoides causing piedra hair infection. Braz J Microbiol. 2012;12:1347-54

18. Guenther E. The essential oils, 1p. 774, Carol stream, IL: D. van Nostrand Co. New York: Princeton York Allured Publication; 1948.

19. Trease GE, Evans MC. Pharmacognosy. 14th ed. New Delhi: Elsevier; 2005.

20. Harborne SB, Baxter H. Phytochemical dictionary: a handbook of bioactive compounds from plants. London: Taylor and Francis; 1995.

21. Burt S. Essential oils: their antibacterial properties and potential applications in foods - a review. Int J Food Microbiol. 2000;94:223-53.

22. Clinical and Laboratory Standards Institute (CLSI). Performance standards for antimicrobial susceptibility testing; twenty-fourth informational supplement. Wayne, USA. 2015.

23. Singh D, Sharma RR. Postharvest disease of fruit and vegetables and their management. In: Prasad D (ed) Sustainable pest management. New Delhi: Daya Publishing House; 2007.

24. lamanaka BT, Taniwaki MH, Menezes HC, Vicente E, Fungaro MHP. Incidence of toxigenic fungi and ochratoxin $A$ in dried fruits sold in Brazil. Food Additives Contaminants. 2005;22:1258-63.

25. Mailafia S, Okoh GR, Olabode HOK, Osanupin R. Isolation and identification of fungi associated with spoilt fruits vended in Gwagwalada market, Abuja, Nigeria. Vet World. 2017;10(4):393-7.

26. Akintobi AO, Okonko IO, Agunbiade SO, Akano OR, Onianwa O. Isolation and identification of fungi associated with the spoilage of some selected fruits in Ibadan, South Western Nigeria. Academ Arena. 2011;3(11):1-10.

27. Tafinta IY, Shehu K, Abdulganiyyu H, Rabe AM, Usman A. Isolation and identification of fungi associated with the spoilage of sweet orange (Citrus sinensis) fruits in Sokoto State. Nigerian J Basic Appl Sci. 2013:21(3):193-6.

28. Chukwuka KS, Okonko IO, Adekunle AA. Microbial ecology of organisms causing Pawpaw (Carica pawpaw L.) fruit decay in Oyo State, Nigeria. American-Eurasian J Toxicol Sci. 2010;2(1):43-50.

29. Pianzzola MJ, Moscatelli M, Vero S. Characterization of Penicillium isolates associated with blue mould on apple in Uruguay. Plant Dis. 2004;88:23-8.

30. Oviasogie FE, Ogofure AG, Beshiru A, Ode JN, Omeje FI. Assessment of fungal pathogens associated with orange spoilage. Afr J Microbiol Res. 2015;9(29):1758-63.

31. Adams MR, Moss MO. Food Microbiology. 3rd ed. North Yorkshire: Royal Society of Chemistry. Athenaeum Press Limited; 1995. p. 130-2.

32. Moss MO. Fungi, quality and safety issues in fresh fruits and vegetables. J Appl Microbiol. 2008;104(5):1239-43.

33. Voysey P. Fruits and vegetables deterioration by fungi. Netherlands: Campden and Charley wood Food Research Association; 2011.

34. Sharma R. Pathogenicity of Aspergillus niger in plants. Cibtech J Microbiol. 2012;1(1):47-51.

35. Chuku EC, Ogbonna DN, Onuegbu BA, Adeleke MTV. Comparative studies on the fungi and biochemical characteristics of snake guard (Trichosanthes curcumerina Linn) and Tomato (Lycopersicon esculentum Mill) in Rivers State, 512 Nigeria. J Appl Sci. 2008;8(1):168-72.
36. Akinmusire OO. Fungal species associated with the spoilage of some edible fruits in Maiduguri, Northern Eastern Nigeria. Adv Environ Biol. 2011:5(1):157-61.

37. Auta Kl, Madinatu B, Yayock HC, Solomon B. Microbial quality assessment of sliced pineapple and watermelon sold in some selected markets in Kaduna metropolis, Kaduna State; 2017.

38. Onuorah S, Orji MU. Fungi associated with the spoilage of postharvest tomato fruits sold in major markets in Awka, Nigeria. Univer J Microbiol Res. 2015;3(2):11-6.

39. Poostforoushfard A, Pishgar AR, Berizi E, Nouraei H, Sobhani Z, Mirzaie $\mathrm{R}$, Zomorodian K. Patulin contamination in apple products marketed in Shiraz, Southern Iran. Curr Med Mycol. 2017:3(4):32-5.

40. Mathew BB, Jatawa SK, Tiwaari A. Phytochemical analysis of Citrus limonum pulp and peel. Int J Pharm Pharm Sci. 2012;4:269-371.

41. Ozcan T, Akpinar-Bayizit A, Yilmaz-Ersan L, Delikanli B. Phenolics in human health. Int J Chem Eng Appl. 2014;5(5):393-5.

42. Bayili RG, Abdoul-Latif F, Kone OH, Diao M, Bassole IHN, Dicko MH. Phenolic compounds and antioxidant activities in some fruits and vegetables from Burkina Faso. Afr J Biotechnol. 2011;10(62):13543-7.

43. Rajput ZI, Hu S, Xiao C, Arijo AG. Adjuvant effects of saponins on animal immune responses. J Zhejiang Univers Sci. 2007;8(3):153-61.

44. Panche AN, Diwan AD, Chandra SR. Flavonoids: an overview. J Nutri Sci. 2016;5:47.

45. Trease GE, Evans WC. Pharmacognosy. Philadelphia: Journal Elsevier Science Limited; 2002.

46. Zanwar AA, Badole SL, Shende PS, Hegde MV, Bodhankar SL. Polyphenols in human health and disease. In: Cardiovascular effects of Hesperidin: a flavanone glycoside, vol. 2. 2014, p. 989-92

47. Tullio V, Nostro A, Mandras N, Dugo P, Banche G, Cannatelli MA, Cuffini AM, Alonzo V, Carlone NA. Antifungal activity of essential oils against filamentous fungi determined by broth microdilution and vapour contact methods. J Appl Microbiol. 2007;102:1544-50.

48. Van HP, Chi PTL, Phi NTL. Comparison of antifungal activities of Vietnamese citrus essential oils. Nat Prod Res. 2013;27:506-8.

49. Madhuri S, Ashwini UH, Srilakshmi NS, Prashith KTR. Antimicrobial activity of Citrus sinensis and Citrus aurantium peel extracts. J Pharm Sci Inn. 2014;3(4):366-7.

50. Amiri H. Chemical composition, antibacterial and antioxidant activity of the essential oil of Tanacetum polycephalum schutz. Bip Intern J Bot. 2007;3:321-4.

51. Jafari S, Esfahani S, Fazeli MR, Jamalifar H, Samadi M, Samadi N, Ardekan MR, Khanavi M. Antimicrobial activity of lime essential oil against foodborne pathogens isolated from cream-filled cakes and pastries. Int J Biol Chem. 2011:5:258-65.

52. Ammad F, Moumena O, Gasem A, Othmane S, Hisashi K, Zebib B, Merah O. The potency of lemon (Citrus limon L.) essential oil to control some fungal diseases of grapevine wood. Comptes Rendus Biologies. 2018;341:97-101.

53. Mello A, Gomes RT, Lara S, Silva G, Alves B. The effect of Brazilian propolis on the germ tube formation and cell wall of Candida albicans. Pharmacol Online. 2006:3:352-8.

54. Budzyńska A, Wieckowska-Szakiel M, Sadowska B, Kalemba D, Rózalska B. Antibiofilm activity of selected plant essential oils and their major components. Polish J Microbiol. 2011;60(1):35-41.

55. Mondello F, De Bernardis F, Girolamo A, Cassone A, Salvatore G. In vivo activity of terpinen-4-ol, the main bioactive component of Melaleuca alternifolia Cheel (tea tree) oil against azole-susceptible and -resistant human pathogenic Candida species. BMC Infect Dis. 2006;6:158.

56. Inouye S, Takizawa T, Yamaguchi H. Antibacterial activity of essential oils and their major constituents against respiratory tract pathogens by gaseous contact. J Antimicr Chemoth. 2001;47:565-73.

57. Sikkema J, de Bont JA, Poolman B. Mechanisms of membrane toxicity of hydrocarbons. Microbiol Mol Biol Rev. 1995;59:201-22.

58. Gallucci MN, Oliva M, Casero C, Dambolena J, Luna A, Zygadlo J, Demo M. Antimicrobial combined action of terpenes against the food-borne microorganisms Escherichia coli, Staphylococcus aureus and Bacillus cereus. Flav Fragr J. 2009;24:348-54.

59. Fisher K, Phillips C. Potential antimicrobial uses of essential oils in food: is Citrus the answer? Trends Food Sci Technol. 2008;19:156-64. 
60. Dinan L, Savchenko T, Whiting P. On the distribution of phytoecdysteroids in plants. Cell Mol Life Sci. 2001;58:1121-32.

61. Wongsariya K, Phanthong P, Bunyapraphatsara N, Srisukh V,

Chomnawang MT. Synergistic interaction and mode of action of Citrus hystrix essential oil against bacteria causing periodontal diseases. Pharma Biol. 2014;52:273-80.

62. Lahlou M. Method to study phytochemistry and bioactivity of essential oil. Phytothe Res. 2004;18:435-48.

63. Lambert RJW, Skandamis PN, Coote PJ, Nychas GJE. A study of the minimum inhibitory concentration and mode of action of Oregano essentia oil, thymol and carvacrol. J Appl Microbiol. 2001;91:453-62.

64. Sharma N, Tripathi A. Effects of Citrus sinensis (L.) Osbeck epicarp essential oil on growth and morphogenesis of Aspergillus niger (L.) Van Tieghem. Microbiol Res. 2008;163:337-44.
65. Caccioni DRL, Guizzardi M, Biondi DM, Renda A, Ruberto G. Relationship between volatile components of citrus fruit essential oils and antimicrobial action of Penicillium digitatum and Penicillium italicum. Intern J Food Microbiol. 1998;43:73-9.

66. Jing L, Lei Z, Li L, Xie R, et al. Antifungal activity of Citrus essential oils: a review. J Agric Food Chem. 2014;62(14):3011-33.

\section{Publisher's Note}

Springer Nature remains neutral with regard to jurisdictional claims in published maps and institutional affiliations.

\section{Submit your manuscript to a SpringerOpen ${ }^{\circ}$ journal and benefit from:}

- Convenient online submission

- Rigorous peer review

- Open access: articles freely available online

- High visibility within the field

- Retaining the copyright to your article

Submit your next manuscript at $\boldsymbol{\nabla}$ springeropen.com 